\title{
Statin related musculoskeletal complications; Necrotizing Autoimmune Myositis... more than Myalgia.
}

\author{
William Scheuing ${ }^{1}$, Dadhania Dadhania ${ }^{1}$, and adegbenga Bankole ${ }^{2}$ \\ ${ }^{1}$ Carilion Clinic \\ ${ }^{2}$ VTCSOM
}

April 20, 2021

\begin{abstract}
Statins are widely prescribed and well tolerated with most side effects now considered a nocebo effect. Occasionally, statins can be associated with immune mediated necrotizing myositis that is both difficult to diagnose and treat. Aggressive immunosuppressive therapy is the best recognized method of treatment of this complication.
\end{abstract}

Statin related musculoskeletal complications; Necrotizing Autoimmune Myositis... more than Myalgia.

William J. Scheuing MD ${ }^{1}$, Frany B. Dadhania $\mathrm{MD}^{1}$, and Adegbenga A. Bankole $\mathrm{MD}^{2}$

1: William J. Scheuing MD \& Frany B. Dadhania MD. Department of Internal Medicine, Virginia Tech Carilion School of Medicine, 2 Riverside Circle, Roanoke, VA 24016, USA.

2: Adegbenga A. Bankole MD. Department of Internal Medicine, Section of Rheumatology, Virginia Tech Carilion School of Medicine, 2 Riverside Circle, Roanoke, VA 24016, USA. Email: aabankole@vt.edu Tel: 540 266-4795 Fax: 540 526-1099 ORCID identifiers: 0000-0001-6464-5367 (Corresponding author)

\section{Introduction:}

Statins significantly reduce the risk of cardiovascular disease and are generally considered safe. On rare occasions, statins can cause muscle disease, and most of these cases recover on discontinuation of the statin. Even more infrequently, statins can cause statin-associated necrotizing autoimmune myositis (SANAM) that is characterized by muscle necrosis on biopsy in the presence of antibodies to 3-hydroxy-3-methylglutaryl coenzyme A (HMG-CoA) reductase. These patients need to be treated with aggressive immunosuppressive therapy, but the treatment response is often poor with a variable clinical response. With the development of newer therapies for dyslipidemia, the prevalence of SANAM as a disease entity will decrease, making it even harder to diagnose and treat. We present a typical case of SANAM with a poor response to aggressive therapy.

Key Clinical Message:

SANAM is difficult to diagnose with some patients presenting after discontinuation of statins. A high degree of suspicion, early referral to rheumatology and aggressive immunosuppressive therapy is required.

Case History:

A 72 year-old man presented to the Emergency Department with a 6-week history of progressive proximal symmetric muscle weakness. He noted some difficulty rising from a seated position, climbing stairs, and 
lifting up his arms to ninety degrees independently. He had no difficulty chewing, talking, swallowing, or opening and closing his eyes. He had diffused joint pain including in his proximal muscle groups in both limb girdles. He had no rash on his face, chest, back, hands, or on his eyelids. One week prior to this presentation, he was diagnosed with left lung basal pneumonia and treated with oral antibiotics. He had fatigue, malaise, night sweats, and dyspnea on exertion. He did not have abdominal pain, change in bowel habits, or black or bloody stools. He did not have dysuria, difficulty voiding, or hematuria. At the time of admission, he was on metoprolol succinate $50 \mathrm{mg}$ daily, furosemide $20 \mathrm{mg}$ daily, and aspirin $81 \mathrm{mg}$ daily. He had been on atorvastatin and sacubitril-valsartan for a number of years, but these medications had been discontinued at the onset of his muscle weakness.

He has paraoesophageal hiatal hernia, Grover's disease, dyslipidemia, hypertension, coronary artery disease, heart failure, and atrial fibrillation. His mother had been diagnosed with dermatomyositis at the age of 72 .

His initial vital signs were normal. He had muscle atrophy in shoulder and hip muscles, but no atrophy was noted in finger flexors. No muscle tremors or fasciculations were observed. His right upper extremity muscle power was $3 / 5$, and $2 / 5$ strength in the left upper extremity. The power in his left and right hip flexors was $2 / 5$. He had $5 / 5$ power in his hands and fingers. His deep tendon reflexes were normal. The nail and nailfold capillaroscopy examination was normal. His joint, pulmonary, and abdominal examinations were normal.

\section{Differential diagnosis, investigations, and treatment:}

The results of his laboratory tests are in Table 1. His blood tests confirmed an elevated creatine kinase (CK) level, as well as elevations in other muscle enzymes also. A bilateral quadricep muscle magnetic resonance imaging (MRI) study was performed (Figure 1), and an MRI directed muscle biopsy was performed (Figure 2a-c). Based on the muscle enzymes levels, positive 3-hydroxy-3-methylglutaryl-coenzyme A reductase antibody (anti-HMGCR Ab) and the results of the muscle biopsy, he was diagnosed with SANAM.

\section{Outcome and follow-up:}

He was started on Prednisone $60 \mathrm{mg}$ per day (approximately $1 \mathrm{mg} / \mathrm{kg}$ ), Methotrexate $20 \mathrm{mg}$ weekly, along with folic acid $1 \mathrm{mg}$ daily, with only a modest improvement in his muscle enzymes and weakness initially. His muscle weakness worsened as the prednisone was tapered. He was readmitted to an in-patient rehabilitation facility and his further changes to his medications. He is currently on a combination of Prednisone, Methotrexate and Rituximab with both improvement in his muscle strength and muscle enzymes levels. He has since been discharged and continues out-patient rehabilitation.

\section{Discussion:}

Statins are very commonly prescribed for dyslipidemia and coronary artery disease. They have antiinflammatory properties and other properties that are beneficial in treatment of a wide range of cardiovascular diseases (5). The side effect profile of statins is very good, with only mild side effects in most cases. Musculoskeletal side effects are among the more commonly reported side effects, but recent studies have shown that most of these complications are a nocebo effect.

Statin use can be associated with SANAM, which is a much more serious complication. Only a small proportion of patients with SANAM improve spontaneously, and even with treatment the outcomes can be poor. With SANAM, prompt and immediate discontinuation of the statin drug is required if the patient is still being treated with it. Following discontinuation of the statin, aggressive immunosuppressive treatment is needed though a clinical response is not always noted. In some cases, the patients continue to deteriorate. There have being no clinical trials on treatment protocols for SANAM and clinical and therapeutic decision is based on case reports, cohort studies and clinical experience and expertise. Oral prednisone at a dose of $1 \mathrm{mg}$ per kilogram of body weight per day is usually the initial therapy, with methotrexate, azathioprine, or mycophenolate mofetil being added as steroid sparing agents (2). Other therapies such as intravenous immune globulin or rituximab may be needed if there is persistent muscle enzyme elevation. Continued muscle weakness may not indicate ongoing muscle disease as fatty replacement of muscle tissue develops and can cause ongoing weakness. 
Our case is a typical presentation as statin-associated necrotizing myopathy and also features its poor response to therapy. SANAM must be considered in the right clinical scenario and if the patients do not respond as expected. Given the uniqueness and specificity of the Anti-HMGCR Ab (6), screening patients with this antibody test may prevent the need for more invasive testing. SANAM is likely to become less common following the development of monoclonal antibody therapies that lower cholesterol. This newer class of medication is highly effective in reduces LDL cholesterol and becoming more commonly prescribed. Given the current ubiquitous use of statins, SANAM is a disease entity that all physicians should be aware of, as early diagnosis allows for early and aggressive treatment that improved the likely outcomes for the patient.

Author Contributions:

William J. Scheuing MD: Performed the literature search, wrote, read, revised manuscript.

Frany B. Dadhania MD: Performed the literature search, wrote, read, revised manuscript.

Adegbenga A. Bankole MD: Performed the literature search, wrote, read, revised and approved the final manuscript.

Conflict of Interest:

No conflict of interests were reported by the authors.

\section{Acknowledgement:}

We thank James W. Mandell, M.D., Ph.D Department of Pathology, University of Virginia School of Medicine, Charlottesville, VA. for aiding with the interpretation of the biopsy slides in this case.

References:

1. Gupta A, Thompson D, Whitehouse A, et al. 2017. Adverse events associated with unblinded, but not with blinded, statin therapy in the Anglo-Scandinavian Cardiac Outcomes Trial-Lipid-Lowering Arm (ASCOT-LLA): a randomised double-blind placebo-controlled trial and its non-randomised non-blind extension phase. Lancet 2017;389:2473-2481.

2. Mammen, Andrew L. 2016. Statin-Associated Autoimmune Myopathy N Engl J Med 2016; 374:664-669

3. Nazir S, Lohani S, Tachamo N, Poudel D, Donato A. 2017. Statin-Associated Autoimmune Myopathy: A Systematic Review of 100 Cases. J Clin Rheumatol. 2017 Apr;23(3):149-154.

4. Gu HM, Zhang DW. 2015. Hypercholesterolemia, low density lipoprotein receptor and proprotein convertase subtilisin/kexin-type 9. J Biomed Res . 2015;29(5):356-361. doi:10.7555/JBR.29.20150067

5. Oesterle A, Liao JK. 2019. The Pleiotropic Effects of Statins - From Coronary Artery Disease and Stroke to Atrial Fibrillation and Ventricular Tachyarrhythmia. Curr Vasc Pharmacol . 2019;17(3):222232. doi: $10.2174 / 1570161116666180817155058$

6. Lucile Musset, Yves Allenbach, Olivier Benveniste, Olivier Boyer, Xavier Bossuyt, et al. 2016. AntiHMGCR antibodies as a biomarker for immune-mediated necrotizing myopathies: A history of statins and experience from a large international multi-center study. Autoimmunity Reviews 15 (2016) 983-993

\section{Hosted file}

Figure 1 MR bilateral lower extremities (femurs) with and without Intravenous Contrast.pdf available at https://authorea.com/users/408903/articles/518723-statin-relatedmusculoskeletal-complications-necrotizing-autoimmune-myositis-more-than-myalgia

Hosted file

Figure 2A Hematoxylin and eosin stain.pdf available at https://authorea.com/users/408903/ articles/518723-statin-related-musculoskeletal-complications-necrotizing-autoimmunemyositis-more-than-myalgia

\section{Hosted file}


Figure 2b Immunostain for type 2 (fast) myosin.pdf available at https://authorea.com/ users/408903/articles/518723-statin-related-musculoskeletal-complications-necrotizingautoimmune-myositis-more-than-myalgia

\section{Hosted file}

Figure 2c Esterase histochemical stain for esterase activity.pdf available at https: //authorea.com/users/408903/articles/518723-statin-related-musculoskeletal-complicationsnecrotizing-autoimmune-myositis-more-than-myalgia

\section{Hosted file}

Table 1.pdf available at https://authorea.com/users/408903/articles/518723-statin-relatedmusculoskeletal-complications-necrotizing-autoimmune-myositis-more-than-myalgia 